\title{
Activités
}

1-1 | avril 2004

Varia

\section{Analyse et modélisation des activités coopératives situées}

Évolutions d'un questionnement et apports à la conception

Pascal Salembier et Bernard Pavard

\section{(2) OpenEdition}

\section{Journals}

Édition électronique

URL : http://journals.openedition.org/activites/1170

DOI : $10.4000 /$ activites. 1170

ISSN : 1765-2723

\section{Éditeur}

ARPACT - Association Recherches et Pratiques sur les ACTivités

\section{Référence électronique}

Pascal Salembier et Bernard Pavard, "Analyse et modélisation des activités coopératives situées », Activités [En ligne], 1-1 | avril 2004, mis en ligne le 02 avril 2004, consulté le 20 avril 2019. URL : http:// journals.openedition.org/activites/1170 ; DOI : 10.4000/activites.1170

\section{(c) $)(9)$}

Activités est mis à disposition selon les termes de la licence Creative Commons Attribution - Pas d'Utilisation Commerciale - Pas de Modification 4.0 International. 


\title{
Analyse et modélisation des activités coopératives situées. Évolutions d'un questionnement et apports à la conception
}

\author{
Pascal Salembier \\ GRIC-IRIT (UMR 5505 CNRS) \\ salembier@irit.fr
}

Bernard Pavard

GRIC-IRIT (UMR 5505 CNRS)

pavard@cict.fr

\begin{abstract}
RÉSUMÉ
Cet article retrace les évolutions d'un programme de recherche centré sur l'analyse, la modélisation et l'instrumentation des activités coopératives. Nous nous attachons notamment à expliciter les changements d'arrièreplan théoriques qui ont guider le déroulement de ce programme et leur influence sur les questions relatives à la modélisation et aux rapports à la conception.
\end{abstract}

MOTS-CLÉ

Activités coopératives, modélisation, conception d'outil coopératifs

\begin{abstract}
This paper summarizes the different steps of a research program which focused on the analysis, modelling and equipement of cooperative activities. We attempt to make explicit the various succesive theorical underpinnings and to show how they have influenced modelling and design issues.
\end{abstract}

KEYWORDS

Cooperative activities, modelling, design of cooperative tools

\section{1.- Introduction}

Le propos de cet article est de repérer quelques problèmes clés apparus dans les recherches menées sur les collectifs de travail et plus particulièrement sur les mécanismes de coopération à travers l'évocation des évolutions d'un programme de recherche théorique, technologique et sociétal mené depuis plus d'une dizaine d'années dans notre laboratoire. Cette évocation ne prétend bien évidemment pas épuiser les questions posées par cette thématique ; elle constitue simplement un fil conducteur pratique permettant d'introduire plusieurs points (explicitation des arrière-plans théoriques, méthodes d'appréhension des objets théoriques ciblés, nature des modèles produits, rapport à la conception des situations) qu'il nous paraît important de considérer dès lors que l'on souhaite contribuer à la réflexion générale sur l'étude des activités professionnelles, collectives et coopératives. 
Evoquer aujourd'hui l'ampleur du développement, au cours des dernières années, des travaux sur les activités collectives et coopératives tient plus de la redondance expressive que de l'apport réel d'information. On peut d'ailleurs se demander rétrospectivement comment on a pu sérieusement penser l'activité comme purement individuelle; sans doute faut-il y voir l'expression de l'influence de modèles issus de disciplines ou de champs théoriques centrés quasi exclusivement sur l'étude de la cognition comme ensemble de processus internes. De notre point de vue une façon pertinente de définir l'activité collective est de se référer à une formulation, inspirée de Jacques Leplat, qui pose l'activité comme « totalité complexe organisée ».

- La notion de totalité renvoie au caractère non décomposable de l'activité ; le choix d'ignorer une dimension de l'activité ne peut être motivé que par des considérations pratiques et en aucune façon, selon nous, par une hypothèse de caractère ontologique portant sur la nature de cet objet.

- La complexité de l'activité renvoie à la multiplicité de ses déterminants qui interagissent de manière dynamique ${ }^{1}$.

- Les modes d'organisation de l'activité sont définis par diverses entités, intrinsèques et extrinsèques, qui opèrent à des niveaux variés (l'organisation, les acteurs, les objets, le contexte, la culture, ...); elles sont en retour « modelées » par l'activité.

L'article est organisé en trois parties qui recouvrent peu ou prou les trois grandes étapes qui ont chronologiquement balisé notre travail ; le choix de cette perspective historique n'est pas dicté par une tendance coupable à l'auto complaisance mais plutôt par le souhait, dans un mouvement de retour sur un passé proche, de pointer les avantages et les limites de différentes orientations théoriques et méthodologiques. Ce jugement doit bien sur être relativisé et rapporté aux critères d'évaluation que nous nous sommes donnés : explicitation claire des présupposés théoriques; articulation entre positionnement ontologique et épistémologique (relation entre objets théoriques et méthodes d'investigation de ces objets); incidences sur la conception des situations. On verra en outre que chacune de ces étapes renvoie plus particulièrement à un thème donné :

- Dans la première partie consacrée à la coopération homme-machine, la question posée est celle des statuts respectifs de l'opérateur humain et de l'artefact avec lequel il interagit dans le cadre d'une activité finalisée conjointe.

- Dans la seconde partie, le principal thème abordé est celui de la construction située d'un référent contextuel partagé entre les membres d'un collectif, et notamment du rôle des mécanismes informels dans la constitution et l'actualisation de ce référent.

- Dans la troisième partie, un des problèmes posés est celui bien connu en sciences sociales de l'articulation de l'individuel et du collectif et des niveaux d'explication micro et macro ${ }^{2}$ : ramener des effets macroscopiques à leurs causes microscopiques n'a en effet rien d'évident dans des situations où la multiplicité des déterminants rend quasi impossible un traçage causal entre comportements individuels et émergence de phénomènes globaux. Les éléments de réflexion, plus que de solution immédiate, qui sont proposés ici s'inspirent de l'application de la théorie de la complexité aux sciences humaines et sociales.

1. Pour une analyse des critères de complexité de l'activité voir Schmidt (2002).

2. Voir par exemple Simmel (1981). 


\section{2.- Etape 1 : la coopération homme-machine et ses limites}

\section{1.- L'illusion mimétique}

Plusieurs conceptions de la coopération homme-machine ont été proposées et défendues depuis une vingtaine d'années. Une première orientation ambitieuse mais également trompeuse jusque dans son évidence métaphorique a consisté à prendre la coopération homme-homme comme unique modèle pertinent et désirable de la coopération homme-machine. Ce positionnement s'est incarné dans des projets visant à développer des systèmes censés incorporer une forme d'intelligence des situations et notamment une part de compréhension des mécanismes régissant l'interaction avec un interlocuteur humain. Sans remettre en cause le pouvoir heuristique de ce positionnement, des voix se sont élevées pour souligner ses limites ; ainsi la reproduction des caractéristiques des situations de coopération homme-homme se heurte à des limitations technologiques, mais également à des problèmes de fond qui ont trait à la non reconnaissance de la profonde asymétrie des partenaires (Suchman, 1987 ; Visetti, 1989). C'est en fait le thème de tout l'ouvrage désormais classique de Lucy Suchman «Plans and Situated Actions» : tous les efforts déployés dans la conception du langage d'interaction et de la dynamique des artefacts informatiques pour masquer cette asymétrie se heurtent rapidement dans les faits à l'irréductibilité des différences entre agents humains et artificiels ${ }^{1}$.

Une approche alternative consiste à intégrer dès le départ cette différence, et à mettre l'accent sur l'identification des « forces » et « faiblesses » supposées de chacun des agents (humain et machine) afin d'allouer à chacun les tâches qui lui conviendront le mieux. Cette posture a donné naissance à une importante somme de travaux sur le thème de «l'allocation de fonction $»^{2}$. D'un point de vue pragmatique l'objectif est ici de fournir au concepteur des éléments de décision, souvent basés sur des modèles naïfs de la performance humaine, qui lui permettent de procéder à la répartition des tâches entre opérateurs et système. Cette démarche, qui présuppose l'existence de compétences spécifiques de l'homme et de la machine selon un mode d'attribution intangible défini une fois pour toute quelle que soit la situation, a trouvé une forme d'achèvement opérationnel dans la production de procédures de type MABA-MABA ( Men Are Better At-Machines Are Better At») pour reprendre une expression de Dekker \& Woods (Dekker \& Woods, 2002). Cette approche a également fait l'objet de nombreuses critiques (voir Dekker \& Woods pour un essai de synthèse) et a donné lieu à tout un champ de recherches qui s'est fixé comme objectif de documenter la singularité des situations de coopération homme-machine autour de la notion de «système cognitif joint » (Woods, Roth, \& Bennett, 1987) où l'artefact informatique d'assistance tient le rôle d'un « outil cognitif » généralement non intrusif et qui reste sous le contrôle de l'opérateur humain.

\section{2.- Une approche pragmatique : l'ingénierie cognitive}

La première période de mise en place de notre programme s'est focalisée sur la conception de systèmes d'assistance coopératifs; elle a adopté une approche fortement influencée par l'ingénierie cognitive telle que développée par Woods et ses collègues (Woods et al., 1987 ; Woods \& Roth, 1988 ; Woods, 1991 ; Woods, Potter, Johannesen, \& Holloway, 1991)

L'unité élémentaire d'analyse en ingénierie cognitive est la « Triade du système cognitif » (Woods, 1988) composée d'un ensemble de trois facteurs qui vont se contraindre mutuellement : le monde, l'agent (humain, machine, système « hybride ») qui opère sur le monde et les instances de médiatisation du réel ou représentations externes via lesquelles l'agent va faire l'expérience du monde.

1. Pour une discussion de quelques problèmes posés par les processus de représentation mutuelle entre agents humains et systèmes d'assistance cf. Cahour \& Salembier (1997).

2. Pour un point récent sur cette problématique, on consultera notamment (McCarthy, Fallon \& Bannon, 2000). 
D'un point de vue technologique l'idée sous-jacente à cette différenciation est que la conception de supports améliorant l'efficacité de la réalisation de la tâche passe nécessairement par la compréhension des interactions entre ces trois éléments. D'un point de vue analytique, la fonction heuristique de ce découpage est de permettre la construction d'une description cognitive autonome de l'environnement (en termes d'exigences et de contraintes notamment) et de faciliter l'identification des facteurs de détermination de la performance.

Dans le «monde » sont particulièrement considérés les facteurs qui vont contribuer à augmenter la complexité cognitive de la tâche à réaliser (nature dynamique de l'environnement, nombre important de parties interconnectées, incertitude des données, risques associés aux conséquences d'une décision inappropriée) ${ }^{1}$. La complexité cognitive peut-être vue ici comme l'interaction entre certaines propriétés de l'environnement et les ressources cognitives de l'agent exprimées en termes de capacités de traitement de l'information qui sont postulées par nature limitées et que l'agent va devoir allouer en fonction de la dynamique des événements de la situation et de son évaluation des mesures à prendre. Les représentations externes vont également affecter la performance en facilitant ou en faisant obstacle à l'accès et à la manipulation de l'information par l'agent.

A ce niveau, le propos de l'ingénierie cognitive est de repérer les configurations conjoncturelles de situations dans lesquelles l'interaction entre les propriétés de ces trois facteurs de détermination de la performance va être susceptible de générer des erreurs. Tout déséquilibre de la Triade qui risque de se traduire par l'émergence d'une situation dangereuse ou non désirée doit alors faire l'objet d'une réponse adaptée en termes de conception d'un outil d'assistance de type «instrument cognitif ». La conception de cet outil passe d'abord par une étape de compréhension de la structure fonctionnelle des tâches du domaine exprimée préférentiellement en termes de buts poursuivis par l'opérateur et de méthodes disponibles pour atteindre ces buts, puis par l'identification d'interactions «triadiques » complexes qui risquent de rendre la réalisation des tâches problématique pour l'agent. On précise alors les tâches qui devront faire l'objet d'une assistance et la forme que prendra cette assistance.

Les méthodes utilisées pour mener à bien le processus de mise en relation systématique entre exigences de la tâche, contraintes imposées par l'environnement et ressources cognitives mobilisables par l'agent, ont été synthétisées sous le terme de « Cognitive Task Analysis » traduite selon les cas ${ }^{2}$ par « analyse de la tâche cognitive » ou « analyse cognitive de la tâche », et qui regroupe un ensemble de méthodes assez diverses, avec une prééminence marquée des méthodes analytiques reposant sur la notion centrale de représentation en buts et en moyens pour atteindre ces buts (goal-means representation). L'outil de base est l'analyse en composants hiérarchiques qui décrit la tâche en buts et sous-buts, et qui associe à chaque élément de la décomposition hiérarchique les moyens disponibles pour les réaliser. Le concept d'espace-problème, bien connu depuis les travaux princeps de Newell et Simon (Newell \& Simon, 1972), constitue l'arrière-plan commun de ces approches, en ce qu'il va permettre d'organiser la description en moyens-buts et fournir un cadre pour identifier les configurations susceptibles de se produire dans le contexte de réalisation de la tâche. Le point important est ici que ces situations sont spécifiées en termes cognitifs et non pas comme en ingénierie classique dans les termes du dispositif technique ; le souci de la démarche est de s'abstraire des particularités propres à une situation singulière et à un système spécifique.

1. Cette analyse proposée par Woods s'inspire directement des travaux de Perrow sur les systèmes critiques (Perrow, 1984)

2. L'interprétation de Cognitive Task Analysis en « analyse de la tâche cognitive » ou " analyse cognitive de la tâche » n'est pas indifférente : la première formulation, la plus neutre, insiste sur la prédominance intellectuelle supposée des tâches analysées : la seconde renvoie à un mode d'analyse spécifique de ces tâches, déterminé par un point de vue particulier sur la nature de la cognition. 
L'approche que nous avons utilisée durant cette étape reprend à son compte plusieurs des positions explicitées par Woods, avec néanmoins la persistance d'une spécificité «culturelle » à savoir le recours systématique à l'analyse de l'activité emblématique de l'ergonomie francophone, là où les modes empiriques d'appréhension du réel utilisés par Woods conservent une part d'ambiguité entre la volonté affichée de s'intéresser à ce que font réellement les opérateurs et le recours quasi systématique à des modèles « classiques » d'analyse de tâches.

Quel est le rôle de cette analyse de l'activité ? Elle est essentiellement de deux ordres :

- un rôle de familiarisation avec le domaine professionnel considéré et avec les pratiques en situation des opérateurs ;

- l'identification de « goulets d'étranglement » (bottlenecks) dans le système, c'est-à-dire de limitations cognitives dans le couplage opérateur-outil (vu comme un "système cognitif joint») et contraintes de l'environnement pointées par l'opérateur.

C'est dire que le passage par l'analyse de l'activité relevait plus pour nous à cette époque d'une nécessité motivée par un choix d'efficacité conceptuelle (au sens de la conception) que d'un projet de fondation de l'activité comme objet théorique singulier.

\section{3.- Statut de la modélisation}

Le rôle des modèles produits au cours de cette étape est essentiellement celui d'un instrument de visibilité et d'intelligibilité. Il s'agit de décrire l'activité des opérateurs confrontés à une tâche complexe et de résumer les dimensions pertinentes de cette activité dans les termes de l'approche de référence (l'ingénierie cognitive). Dans un premier temps un modèle de la tâche réelle (i.e. les objectifs assignés par l'organisation tels qu'ils sont reformulés par l'opérateur) est construit sur la base d'entretiens menés avec les opérateurs (ici des régulateurs de la navigation aérienne). Dans un second temps l'activité de l'opérateur est analysée en situation et décrite en termes de mécanismes cognitifs de haut niveau ${ }^{1}$ (diagnostic, planification, résolution de problèmes, ...). Pour chaque étape ou dimension de l'activité on pointe les goulets d'étranglement en termes de limitations du couplage capacités cognitives de l'opérateur et exigences de gestion des situations, définies par l'interaction entre tâches à réaliser et incertitudes liées au caractère complexe de l'environnement (Pavard \& Salembier, 1991). La relation avec la conception est ici directe : l'identification des limitations doit permettre la spécification d'outils cognitifs et de fonctionnalités permettant de les dépasser (Salembier, 1994). À noter ici qu'à la différence d'autres approches étiquetées « Ingénierie cognitive », le repérage de problème de couplage est effectué sur la base de l'analyse de l'activité en situation et non pas à partir de la description de la tâche prescrite ou réelle.

\section{4.- Les limites de la démarche}

Une première limitation de cette approche de la coopération tient à sa centration sur l'activité d'un sujet, isolé artificiellement dans sa relation exclusive à un artefact cognitif ${ }^{2}$. L'analyse de la coopération se restreint à l'étude de l'interaction homme-machine, et la dimension collective de l'activité n'est au mieux envisagée que par le biais de la reconnaissance implicite de l'incorporation de l'agent dans un système organisationnel plus large et au travers d'éventuelles communications verbales interpersonnelles.

1. Par opposition aux mécanismes dit de « bas niveau », encapsulés et inaccessibles à la conscience du sujet pensant et agissant.

2. Sur ce point comme sur d'autres la position de Woods a considérablement évolué. 
Une seconde limitation vient du fait que l'accent est mis sur le couplage entre les capacités de traitement de l'information de l'opérateur et le monde extérieur via la médiation d'un système de représentation symbolique ${ }^{1}$ (panneau de contrôle, écran d'ordinateur, ...). Les propriétés fonctionnelles des artefacts (déterminées notamment par leur caractéristiques physiques) ne sont pas considérées. On a donc au final l'image réductrice d'une cognition individuelle, désincarnée, où l'opérateur est vu essentiellement sous l'angle d'un système qui alloue des ressources cognitives limitées pour répondre aux exigences de la tâche et aux fluctuations de l'environnement.

\section{3.- Etape 2 : mécanismes informels de la coopération homme- homme}

Le rôle des collectifs de travail dans la redéfinition des tâches assignées par l'organisation est une question qui a été amplement documentée dans plusieurs études (cf. par exemple Terssac, 1992). Ce processus de redéfinition se manifeste par la constitution de règles non écrites, informelles, non prescrites et donc souvent ignorées de l'organisation mais qui jouent un rôle fondamental dans la réalisation satisfaisante (pour les opérateurs et l'organisation) des tâches.

Au cours de cette seconde étape, et en réponse à une exigence des situations de travail sur lesquelles nous intervenions ${ }^{2}$, nous avons été amenés à nous centrer sur un aspect particulier de ces mécanismes informels : la construction et l'actualisation dans le cours de l'activité d'un référentiel contextuel partagé qui constitue une des conditions du déploiement efficace de l'activité collective dans un environnement complexe distribué et la régulation par ce collectif de facteurs tels que les variations dans la charge de travail et la fiabilité globale du système socio-technique. Les travaux menés au cours de cette étape ont été essentiellement conduits dans deux domaines : la régulation des appels d'urgence (centre SAMU) et le contrôle du trafic aérien.

\section{1.- La centration sur le collectif}

A la différence de l'étape précédente, l'accent est mis ici sur la coopération ${ }^{3}$ entre agents humains, mais sans faire l'erreur consistant à ignorer les artefacts (cognitifs ou non).

Cette nouvelle orientation répondait ici à une double motivation :

- la volonté de traiter un aspect repoussé consciemment jusqu'alors dans l'arrière-plan d'activités individuelles s'entrecroisant et se coordonnant parfois ;

- la nécessité de répondre à de nouveaux problèmes de conception posés en termes de supports informatiques à la coopération entre opérateurs, dans la dynamique instaurée par le courant CSCW (Computer Supported Cooperative Work).

L'analyse de l'activité joue toujours ici un rôle structurant majeur avec une importance particulière donnée aux aspects non directement liés à la tâche mais à l'obtention des conditions efficaces de sa réalisation (anticipation des actions des autres agents, constitution d'une représentation contextuelle partagée, ...). La difficulté est ici de parvenir à identifier et à décrire des mécanismes inséparables de leur contexte de production, non connus et reconnus par l'organisation et souvent difficilement conscientisables par les acteurs eux-mêmes.

1. Ceci s'explique en partie par le type de situation majoritairement investiguée (salles de contrôle dans le nucléaire, cockpit d'avion).

2. Exigences mises en évidence par des campagnes prolongées d'analyse de l'activité dans deux domaines : la régulation des appels d'urgence (centre SAMU) et le contrôle du trafic aérien.

3. Pour une définition précise de ce que nous entendons par le terme de coopération, voir Salembier \& Zouinar (2000); Zouinar (2000). 


\section{2.- La décentration relative d'avec le domaine de tâches}

Poursuivant en cela une tradition bien établie en ergonomie francophone, et à la différence des modèles de la coopération que l'on qualifiera de « classiques ", souvent d'inspiration cognitiviste (Zachary \& Robertson, 1990), le travail mené au cours de l'étape 2 met l'accent sur l'élucidation des mécanismes de régulation du collectif, et plus particulièrement des mécanismes informels de cette régulation. L'objectif est ici de tenter de mettre en lumière la part de l'activité collective non directement liée ou exprimable dans les termes du domaine de tâche, méconnue la plupart du temps de l'organisation, souvent non conscientisée spontanément par les opérateurs eux-mêmes, mais qui est néanmoins indispensable à la réalisation satisfaisante de cette tâche. Ceci nécessite donc la mise en œuvre d'un modèle d'analyse qui n'exprime plus uniquement l'activité dans les termes du domaine de tâches (même dans le cas où celui-ci est exprimé par des catégories descriptives d'un certain niveau d'abstraction : diagnostic, supervision, ...). Cette orientation est proche dans l'esprit des études menées dans le courant du CSCW (Computer Supported Cooperative Work) notamment par la sociologie d'inspiration ethnométhodologique, qui partage sur l'étude des situations de travail certaines positions avec l'ergonomie de langue française (voir par exemple les travaux menés par Heath et ses collègues (Heath \& Luff, 2000). Il reste néanmoins, et ce à la différence de certains travaux menés en ethnométhodologie, que l'ancrage dans la spécificité du domaine professionnel investigué reste pour nous une nécessité : réduire une situation de travail à une occasion de recueil de données permettant de documenter des mécanismes généraux de régulation du collectif sans considérer les objectifs spécifiques poursuivis par les opérateurs et en perdant de vue que l'analyse empirique s'inscrit dans une démarche de conception / transformation, ne constitue pas pour nous une issue viable.

\section{3.- Le rôle des artefacts}

Bien que prenant en compte le rôle des supports d'informations et leurs éventuelles limitations, en termes de facilité d'accès et de traitement notamment, les analyses menées lors de la première étape restaient assez superficielles quant aux propriétés physiques des objets utilisés par les opérateurs. Ainsi les objets étaient essentiellement considérés dans leur dimension informationnelle (artefacts cognitifs) et non dans leur dimension manipulable pour reprendre la distinction bien connue introduite par Norman (Norman, 1991). Cette ignorance de la constitutivité matérielle des activités cognitives, notamment collectives, faisait inconsciemment écho aux positions du progamme cognitiviste $^{1}$.

Cette nouvelle importance donnée aux propriétés physiques des objets matériels et à leur relation avec la cognition individuelle et collective ne faisait en fait que rejoindre des préoccupations antérieures sur la nature de la relation entre représentations internes et externes déjà manifestées en ergonomie (Pavard, 1985 ; Pavard, Marmaras, \& Xanthoudakis, 1987) en anthropologie cognitive (Hutchins, 1995) et en sociologie des techniques (Latour, 1992) et bien thématisées par Schmidt (Schmidt, 1999) dans le contexte du CSCW.

Dans les situations professionnelles auxquelles nous nous sommes intéressés, un des exemples quasi paradigmatiques de ce processus d'externalisation de la cognition dans des objets matériels porte sur l'analyse de l'utilisation du «strip » (bande de papier utilisée par les contrôleurs du trafic aérien pour gérer les vols dont ils ont la responsabilité). Cet artefact se caractérise par ses propriétés informationnelles : on peut y reporter des informations relatives aux instructions données aux vols : valeurs de cap, niveau, vitesse, ... ainsi que d'autres formes d'annotation. Il se caractérise également

1. Qui outre les aspects matériels a ignoré sciemment pendant longtemps les dimensions corporelles, émotionnelles et culturelles de la cognition. 
par ses propriétés physiques de manipulabilité qui autorisent l'agencement de configurations spatiales signifiantes pour le contrôleur.

Outre leur statut de support à l'activité individuelle (mémorisation, planification), les strips en tant que représentations publiques jouent également un rôle important dans la coordination entre contrôleurs, en permettant notamment la reconnaissance d'intention et l'actualisation d'un contexte partagé nécessaire à la réalisation collective de la tâche (Benchekroun, Pavard, \& Salembier, 1994 ; Bressolle, 1993 ; Salembier, 2002).

\section{4.- Statut de la modélisation}

\subsection{1.- Construction des modèles}

Une des spécificités de notre mode d'appréhension des activités collectives tient à la place donnée à la modélisation. Alors que par exemple la construction de modèles n'est pas jugée nécessaire voire utile par les ethnométhodologues (cf. Anderson, 1997), la modélisation dans cette étape de nos travaux passe du simple statut à vocation descriptive à celui de support à la simulation. Les modèles développés sont donc exprimés avec un système de notation formel basé sur la logique des prédicats, sans que l'on considère qu'il y ait nécessaire isomorphisme entre activités cognitives et procédures logiques d'expression de certains aspects de ces activités. Ce type de modèle constitue ce que Theureau (Theureau, 2002) a dénommé à la suite d'une analyse de Timmermans inspirée de Spinoza un modèle de type «empirique synthétique ». La finalité est ici de disposer d'un modèle centré sur un aspect particulier de l'activité collective (dans le cas qui nous occupe il s'agit des processus de constitution et d'actualisation du contexte partagé), qui repose sur une base empirique étoffée et qui d'un point de vue opérationnel puisse permettre d'orienter des décisions de transformation des situations de travail. Cette orientation nécessite que le modèle soit suffisamment indépendant des spécificités d'une situation, ce qui conduit donc à opérer des simplifications (éventail des événements susceptibles de se produire dans la situation donnée) qui limitent le domaine de validité a priori du modèle. Ainsi dans le cas de la modélisation du contexte partagé, on s'exprime en termes d'informations potentiellement partagées dans la mesure où la réalité effective de ce partage est largement indécidable.

Cet intérêt particulier pour les processus de constitution du contexte partagé tient à leur incidence sur la gestion collective de la fiabilité du système sociotechnique par la mise en place de différents mécanismes de régulation basés notamment sur les modes informels de gestion du collectif. Cet aspect de notre travail a donné lieu à la production d'un modèle basé sur la notion de «boucles de régulation » (Bressolle, Decortis, Pavard, \& Salembier, 1996; Rognin, Salembier, \& Zouinar, 2000). Il s'agit à nouveau ici comme dans l'étape 1 d'une version stylisée de la réalité qui néglige volontairement certains éléments afin d'en faire ressortir d'autres ${ }^{1}$. Dans le même temps on pourrait dire que l'accent mis ici sur la relation entre activité coopérative (contexte partagé, contrôle mutuel des actions, régulation collective de la charge de travail,...) et phénomène émergent au niveau du système lui-même (fiabilité du contrôle aérien) instaure une transition avec la phase 3 (Salembier \& Zouinar, 2000).

\subsection{2.- De la modélisation à la simulation}

Pourquoi recourir à une étape de simulation? Il convient tout d'abord de préciser ce que nous entendons par ce terme. À la différence des entreprises de simulation dite cognitive engagées durant les

1. Cette orientation sélective propre à toute entreprise de modélisation a conduit certains auteurs à définir la construction de modèles comme l'expression d'une « stratégie de la négligence » (Nouvel, 2002). 
années 80 avec un certain optimisme non dénué de naïveté et issues de la branche technologique du programme cognitiviste, i.e. l'intelligence artificielle, l'objectif n'est pas ici de reproduire le fonctionnement du système cognitif ni de prédire de façon précise les sorties de ce système. Nos simulations sont prédictives mais dans un sens beaucoup plus faible que celui utilisé classiquement, en tant que moyen d'administration de la preuve par exemple. L'idée est ici plutôt de jouer sur un ensemble de variables exogènes d'environnement pour explorer un changement de situation (introduction d'un nouvel outil, modifications des formes d'organisation du collectif, ...) et évaluer son impact sur des variables endogènes jugées pertinentes -par exemple une appréciation quantitative du contexte partagé- (Zorola-Villarreal, Pavard, \& Bastide, 1995 ; Salembier, Kahn, Zorola-Villarreal, \& Zouinar, 1997). La simulation fonctionne comme un moyen pour « ouvrir l'espace de conception » et nourrir l'interaction (discussion et négociation) entre les acteurs engagés dans la conception autour de différentes alternatives possibles.

\section{5.- Les limites}

Une des limites de cette approche outre une relative lourdeur de mise en œuvre lorsqu'on souhaite aller au bout de la démarche tient à la nature déterministe de la simulation : la modélisation et la simulation qui la réalise physiquement fonctionnent comme des machines à calculer symboliques qui permettent d'aller beaucoup plus vite et de manière plus fiable que lors d'une simulation papiercrayon, mais en contrepartie on est rarement « surpris » par les sorties obtenues. L'intérêt est donc de ce point de vue relatif, bien que ceci puisse être retourné en argument positif dans la mesure où le sens et la signification des résultats restent intelligibles. Par ailleurs le gain doit également être évalué en intégrant les effets dus à l'exigence supplémentaire de rigueur dans la description et la notation des données, et ceux inhérents à la fonction argumentative de la simulation.

\section{4.- Etape 3 : activités complexes et modèles de la complexité}

\section{1.- Autonomie du modèle ?}

Le passage à l'étape 3 constitue du point de vue de la modélisation et surtout de la simulation un changement de paradigme drastique dans la mesure où il nous fait passer des outils logiques classiquement utilisés en sciences cognitives aux théories de la complexité et aux systèmes dynamiques non linéaires (Pavard, 2002). Cette nouvelle orientation s'inscrit dans le contexte plus général du développement des outils de simulation en sciences sociales qui fournissent des moyens de prédiction et surtout de simulation dynamique permettant d'anticiper l'émergence de faits sociaux par les comportement individuels d'agents ${ }^{1}$. La raison qui motive ce choix tient à la reconnaissance et à l'acceptation du fait que la dynamique des processus qui supportent la coopération et qui sont à l'œuvre dans les situations professionnelles sont la plupart du temps impossibles à prévoir car non déterministes du fait notamment de leur caractère distribué et de leur sensibilité aux variations environnementales (Pavard \& Dugdale, 2000).

D'un point de vue global la démarche mise en œuvre s'éloigne peu de celle utilisée durant la seconde étape (Dugdale \& Pavard, 2000). On retrouve sous une forme élargie les outils classiques utilisés antérieurement : analyse de la tâche, analyse de l'activité, simulation papier-crayon, simulation partielle de la situation, mise en situation recréée, simulation informatique.

Par contre du point de vue de la modélisation, on rentre dans une toute autre dimension de par les capacités d'autonomie qui sont allouées aux agents. On se trouve ainsi dans le cas d'une modélisa-

1. Voir par exemple les travaux menés dans le cadre du réseau européen COSI (http://www.irit.fr/COSI). 
tion « dramatique ${ }^{1}$ » dans la mesure où « on met sur scène les protagonistes » dotés ici d'un répertoire comportemental limité mais qui permet l'émergence de propriétés globales par le biais des interactions entre ces agents.

Le choix qui est fait ici est de limiter la finesse de la modélisation de l'activité individuelle et de privilégier la modélisation des processus collectifs. Mais cette option, qui repose sur le constat d'imprédictibilité des comportements des agents, n'a de sens que si l'on se dote par ailleurs d'outils de simulation permettant d'explorer les évolutions possibles du système sociotechnique en réponse à différentes conditions de départ initiales (organisation de l'espace particulière, modalités d'organisation du travail dans le collectif, introduction de nouveaux outils coopératifs,...). Ceci passe par le recours à des outils de simulation particuliers qui se distinguent de ceux utilisés dans l'étape 2.

\section{2.- Statut de la simulation}

Les outils utilisés sont essentiellement des environnements de simulation multi-agents qui permettent de recréer la dynamique du fonctionnement du collectif en réponse à des modifications de l'environnement externe (survenue d'événements particuliers) ou interne (modification de la structure du collectif d'agents). Chaque agent est décrit par un ensemble d'attributs qui vont définir un éventail de comportements possibles ; ces attributs sont soit prédéfinis, soit générés dynamiquement au cours de la simulation.

A la différence du type de simulation menée dans l'étape 2, on peut théoriquement s'attendre ici à obtenir des résultats non attendus, voire même «surprenants ». Le problème est de savoir quel niveau de confiance on peut leur accorder et comment les utiliser. Va-t-on les considérer comme des éléments heuristiques de réflexion, comme des outils d'aide à l'interprétation a posteriori de phénomènes particuliers, ou comme des outils permettant de s'approcher de la prédiction de ce qui risque de se passer? Dans la manière dont est envisagé le statut de la simulation dans nos travaux, il s'agit essentiellement de disposer d'éléments d'appréciation du rôle de certains mécanismes sur un phénomène plus global (Dugdale \& Pavard, 2000). La validation ne se fait pas sur les comportements locaux des agents mais sur la capacité du modèle à faire émerger des propriétés globales du système intéressantes qui pourront contribuer à enrichir le processus de conception.

\section{3.- Limites}

Une première limite de ce type de modélisation tient à la fascination que peut exercer la possibilité de multiplier de façon relativement commode des simulations qui finalement n'apportent pas de véritable enseignement : soit parce que les résultats produits sont difficilement interprétables, soit parce qu'ils sont triviaux (i.e. anticipables sans recours à une simulation), soit parce que le répertoire d'actions des agents est si limité que le modèle n'a plus grand chose à voir avec la réalité (ce qui pose un problème dans la mesure où une modification légère dans le comportement d'un agent peut générer des modifications importantes à l'échelle du système). Le risque est donc de se concentrer essentiellement sur l'émergence de propriétés globales sans pouvoir les articuler avec les comportements locaux des agents ; le spectre d'un collectivisme méthodologique radical n'est pas loin... On ne doit pas en outre occulter le coût de ce type de modélisation et des simulations associées, alors même que leur utilité effective pour le processus de conception demeure questionnable, et ce y compris dans des situations où les risques (humains et matériels) justifient la mise en œuvre de moyens importants.

Une seconde limite tient à l'usage même des systèmes multi-agents qui imposent une modélisation souvent élémentaire des propriétés sociales et cognitives des acteurs humains qu'ils sont censés

1. La distinction entre modélisation dramatique et modélisation mathématique a été introduite par B. Saint-Sernin. 
représenter. La dimension "située » des acteurs, leurs connaissances culturelles, leur intelligence contextuelle, ... sont des dimensions difficiles sinon impossibles à représenter de façon synthétique dans les agents distribués (Dugdale \& Pavard, 2002). C'est pour dépasser ces contraintes que nous nous sommes récemment orientés vers des paradigmes de simulation permettant de bénéficier à la fois des propriétés d'émergence propres aux systèmes distribués et des propriétés de mise en situation grâce à l'usage de la réalité virtuelle (Darcy, Dugdale, Mehdi, Pallamin, \& Pavard, soumis). Dans cette approche, les agents humains évoluent et interagissent entre eux et avec leur environnement dans un univers virtuel. Ils sont ainsi «en situation » et peuvent prendre des décisions en se basant aussi bien sur les artefacts de leur environnement que sur leurs acquis cognitifs et culturels.

\section{5.- Conclusion}

S'il fallait se risquer à un commentaire de synthèse pour une telle rétrospective, peut-être pourraiton dire que le moteur de cette recherche a été un souci permanent de trouver les cadres conceptuels adaptés à la problématique de l'analyse et de la conception d'environnements socio-techniques complexes. Etant issus de la culture analytique classique, qui a fortement structuré le champ de la recherche en ergonomie et en science cognitive, il nous a fallu y échapper en se dotant d'outils de simulation nous permettant de passer d'une approche structurelle et descriptive (post hoc) à une approche productive capable de modéliser des comportements collectifs et en particulier leurs propriétés d'émergence. Nous avons pour cela largement fait appel à des champs disciplinaires connexes (théorie des actes de langage, théorie des systèmes complexes, éthologie, théorie des systèmes multi-agents, interactionnisme, ...). Le véritable changement paradigmatique est survenu avec l'appropriation du concept d'émergence (ou d'auto organisation) qui nous a permis de resituer notre recherche dans le cadre d'une épistémologie plus constructiviste tout en gardant la rigueur de la démonstration grâce à l'usage des modèles de production. Restait à traiter dans ce contexte le problème du caractère situé des interactions humaines (qu'elles soient cognitives ou culturelles). Nous abordons aujourd'hui ce challenge avec nos collègues ethnométhodologues et spécialistes de la réalité virtuelle. Nous pensons que ce nouvel axe de recherche nous amènera à mieux comprendre et penser les situations de travail collectif de demain.

\section{REMERCIEMENTS}

Cette note de synthèse est l'expression d'un travail collectif mené sur plusieurs années et qui a impliqué plusieurs membres présents et passés du laboratoire GRIC-IRIT ; nous citerons notamment : Haleh Ashtiani, Marie-Christine Bressolle, Sandrine Darcy, Julie Dugdale, Julien Kahn, Laurence Rognin, Renaut Zorolla, Moustapha Zouinar.

Merci à Marianne Cerf et Alain Lancry pour leur relecture de cet article et pour la pertinence de leurs remarques.

\section{BIBLIOGRAPHIE}

Anderson, J. R. (1997). Work, ethnography, and system design. In A. Kent \& J. G. Williams (Eds.), The encyclopedia of Microcomputing (Vol. 20, pp. 159-183). New York: Marcel Dekker.

Benchekroun, T. H., Pavard, B., \& Salembier, P. (1994). Design of cooperative systems in complex dynamic environments. In J. M. Hoc, C. Cacciabue \& E. Hollnagell (Eds.), Expertise and Technology - Cognition \& Human-Computer Cooperation (pp. 167-182). New Jersey: LEA.

Bressolle, M. C. (1993). Contribution à l'étude de la perception de l'intention et de la coopération. Le cas des contrôleurs de la navigation aérienne dans le survol national. Unpublished Mémoire de DEA, Université de Toulouse le Mirail, Toulouse. 
Bressolle, M. C., Decortis, F., Pavard, B., \& Salembier, P. (1996). Traitement Cognitif et Organisationnel des micro-incidents dans le domaine du contrôle du trafic aérien : Analyse des boucles de régulation formelles et informelles. In G. de Terssac \& E. Friedberg (Eds.), Coopération et Conception. Toulouse: Octares Editions.

Cahour, B., \& Salembier, P. (1997). Cooperation and Cooperator Modelling. Computer-Supported Cooperative Work Journal(1).

Darcy, S., Dugdale, J., Mehdi, E. J., Pallamin, N., \& Pavard, B. (soumis). Virtual reality, story building, story telling.

Dekker, S. W. A., \& Woods, D. D. (2002). MABA-MABA or Abracadabra? Progress on Human-Automation co-ordination. Cognition, Technology \& Work(4), 240-244.

Dugdale, J., \& Pavard, B. (2000). The Application of a Cognitive Engineering Methodology to Agent-based Simulation in the Social Sciences. In Proceedings of Agent-Based Simulation Workshop 2000. May 2-3, Passau, Germany.

Dugdale, J., \& Pavard, B. (2002). From representational to contextual intelligence. Paper presented at the Computational and Mathematical Organization Theory Conference, Pittsburgh, USA.

Heath, C., \& Luff, P. (2000). Technology in action. Cambrige: Cambridge University Press.

Hutchins, E. (1995). Cognition in the wild: Bradford Books-MIT Press.

Latour, B. (1992). Where are the missing masses, Sociology of a few mundane artefacts. In W. Bijker \& J. Law (Eds.), Shaping Technology-Building societies. Studies in sociotechnical change (pp. 225-259). Cambridge MA: MIT Press.

McCarthy, J., Fallon, E., \& Bannon, L. (2000). Dialogues on function allocation. Int. J. Human-Computer Studies(52), 191-201.

Newell, A., \& Simon, H. A. (1972). Human problem-solving. Englewood Cliffs, N.J : Prentice Hall.

Norman, D. A. (1991). Cognitive artefacts. In J. M. Caroll (Ed.), Designing interaction psychology at the human-computer interface. Cambridge: Cambridge University Press.

Nouvel, P. (2002). Modèles et métaphores. In P. Nouvel (Ed.), Enquête sur le concept de modèle (pp. 189202). Paris : PUF.

Pavard, B. (1985). Editeurs de texte et stratégies de rédaction de dépêche: analyse des contraintes pragmatiques. Intellectica, 1(1), 37-68.

Pavard, B. (2002). Complexity paradigm as a framework for the study of cooperative systems. In P. Salembier \& T. H. Benchekroun (Eds.), Cooperation and complexity. Paris-Londres : RSTI-RIA, Vol. $16 \mathrm{n}^{\circ}$ 4-5, Hermes-Lavoisier.

Pavard, B., \& Dugdale, J. (2000). The contribution of complexity theory to the study of sociotechnical systems. New England Complex Systems Institute electronic journal,. http://www.interjournal.org.

Pavard, B., Marmaras, N., \& Xanthoudakis, H. (1987). Les changements de représentation induits par les sytèmes informatriques. Revue de l'Académie des Sciences de l'U.R.S.S, 8(1).

Pavard, B., \& Salembier, P. (1991). L'aide à la régulation du trafic aérien : analyse de l'activité et assistance coopérative à la décision (No. Rapport convention NEB/CENA). Paris : CNAM.

Perrow, C. (1984). Normal accidents : Living with high-risk technologies. New-York : Basic Books.

Rognin, L., Salembier, P., \& Zouinar, M. (2000). Cooperation, reliability of socio-technical systems and allocation of function. International Journal of Human-computer Studies, 52(2), 357-379.

Salembier, P. (1994). Assistance coopérative aux activités complexes : l'exemple de la régulation du trafic aérien. In B. Pavard (Ed.), Systèmes coopératifs : de la modélisation à la conception. Toulouse : Octares.

Salembier, P. (2002). Cadres conceptuels et méthodologiques pour l'analyse, la modélisation et l'instrumentation des activités coopératives situées. Systèmes d'information et Management (SIM), $n^{\circ} 2$, Vol. 7, 37-56.

Salembier, P., Kahn, J., Zorola-Villarreal, R., \& Zouinar, M. (1997). Assessing of methods : Cognitive modeling (No. WP6, RHEA.) : CEC, DG VII. 
Salembier, P., \& Zouinar, M. (2000). Analysing and modelling mutual awareness in cooperative work settings, http://www-sv.cict.fr/cotcos/pjs/

Schmidt, K. (1999). Of maps and scripts : the status of formal constructs in cooperative work. Information and Software Technology, 41, 319-329.

Schmidt, K. (2002). Remarks on the complexity of the cooperative work. In P. Salembier \& T. H. Benchekroun (Eds.), Cooperation and Complexity, RSTIA. Paris-London : Hermes-Lavoisier.

Simmel, G. (1981). Sociologie et épistémologie. Paris: PUF.

Suchman, L. A. (1987). Plans and situated actions - The problem of human-machine communication. Cambridge: Cambridge University Press.

Terssac, G. de. (1992). Autonomie dans le travail. Paris: PUF.

Theureau, J. (2002). Dynamic, living, social and cultural complex systems : principles of design-oriented analysis. In P. Salembier \& T. H. Benchekroun (Eds.), Cooperation \& Complexity, RSTIA. Paris-Londres: Hermes-Lavoisier.

Visetti, Y.-M. (1989). Compte rendu de Lucy A. Suchman, Plans and Situated Actions - The problem of Human/Machine Communication. Intellectica (9).

Woods, D. D. (1988). Coping with complexity: the psychology of human behavior in complex systems. In L. P. Goodstein, H. B. Andersen \& S. E. Olsen (Eds.), Tasks, Errors and Mental Models (pp. 128-148). London: Taylor and Françis.

Woods, D. D. (1991). The cognitive engineering of problem representations. In G. R. S. Weir \& J. L. Alty (Eds.), Human-computer Interaction and complex systems. Londres: Academic Press.

Woods, D. D., Potter, S. S., Johannesen, L., \& Holloway, M. (1991). Human interaction with intelligent systems : Volume I - Trends, problems, new directions (CSEL Report No. 1991-001). Columbus OH: Ohio State University.

Woods, D. D., \& Roth, E. M. (1988). Cognitive systems engineering. In M. Helander (Ed.), Handbook of Human Computer Interaction. North Holland: Elsevier Science Publishers B.V.

Woods, D. D., Roth, E. M., \& Bennett, K. (1987). Explorations in joint human-machine cognitive systems. In W. Zachary \& S. Robertson (Eds.), Cognition, computing and cooperation. Norwood NJ: Ablex.

Zachary, W., \& Robertson, S. P. (1990). Introduction to cognition, computation, and cooperation. In S. P. Robertson, W. Zachary \& J. B. Black (Eds.), Cognition, computing, and cooperation. Norwood, New Jersey: Ablex Pub. Corp.

Zorola-Villarreal, R., Pavard, B., \& Bastide, R. (1995). SIM'COOP : A tool to analyse and predict cooperation in complex environments. Paper presented at the Fifth International Conference on HumanMachine Interaction \& Artificial Intelligence in Aerospace, September, Toulouse, France.

Zouinar, M. (2000). Analyse et modélisation des processus de construction et d'actualisation du contexte partagé. Unpublished Thèse de Doctorat, CNAM, Paris. 